\title{
Independent Multimodal Background Subtraction
}

\author{
Domenico Bloisi and Luca Iocchi \\ Department of Computer, Control, and Management Engineering - Sapienza University of Rome, Italy
}

Background subtraction is a common method for detecting moving objects from static cameras able to achieve real-time performance. However, it is highly dependent on a good background model particularly to deal with dynamic scenes. In this paper a novel real-time algorithm for creating a robust and multimodal background model is presented. The proposed approach is based on an on-line clustering algorithm to create the model and on a novel conditional update mechanism that allows for obtaining an accurate foreground mask. A quantitative comparison of the algorithm with several state-of-the-art methods on a well-known benchmark dataset is provided demonstrating the effectiveness of the approach.

\section{INTRODUCTION}

Background subtraction (BS) is a popular method for detecting moving objects from static cameras able to achieve real-time performance. BS aims to identify moving regions in image sequences comparing the current frame to a model of the scene background (BG). The creation of such a model is a challenging task due to illumination changes (gradual and sudden), shadows, camera jitter, movement of background elements (e.g., trees swaying in the breeze, waves in water), and changes in the background geometry (e.g., parked cars).

Different classifications of BS methods have been proposed in literature. In (Cristani and Murino 2008), BS algorithms are organized in: 1) per pixel, 2) per region, 3) per frame and 4) hybrid. Per-pixel approaches (e.g., (Cucchiara et al. 2003; Stauffer and Grimson 1999)) consider each pixel signal as an independent process. Per-region algorithms (e.g., (Heikkila and Pietikainen 2006)) usually divide the frames into blocks and calculate block-specific features in order to obtain the foreground. Frame-level methods look for global changes in the scene (e.g., (Oliver et al. 2000)). Hybrid methods (e.g., (Wang and Suter 2006; Toyama et al. 1999)) combine the previous approaches in a multi-stage process.

In (Cheung and Kamath 2004) two classes of BS methods, namely recursive and non-recursive, are identified. Recursive algorithms (e.g., (Stauffer and Grimson 1999)) maintain a single background model that is updated with each new input frame. Nonrecursive approaches (e.g., (Cucchiara et al. 2003; Oliver et al. 2000)) maintain a buffer of previous video frames and estimate the background model based on a statistical analysis of these frames.

A third classification (e.g., (Mittal and Paragios 2004)) divides existing BS methods in predictive and non-predictive. Predictive algorithms (e.g., (Doretto et al. 2003)) model the scene as a time series and develop a dynamical model to recover the current input based on past observations. Non-predictive techniques (e.g., (Stauffer and Grimson 1999; Elgammal et al. 2000)) neglect the order of the input observations and build a probabilistic representation of the observations at a particular pixel.

Although all the above mentioned approaches can deal with dynamic background, a real-time, complete, and effective solution does not yet exist. In particular, water background is more difficult than other kinds of dynamic background since waves in water do not belong to the foreground even though they involve motion. Per-pixel approaches (e.g., (Stauffer and Grimson 1999)) typically fail because these dynamic textures cause large changes at an individual pixel level (see Fig. 1) (Dalley et al. 2008). A nonparametric approach (e.g., (Elgammal et al. 2000)) is not able to learn all the changes, since in the water surface the changes do not present any regular patterns (Tavakkoli and Bebis 2006). More complex approaches (e.g., (Sheikh and Shah 2005; Zhong and Sclaroff 2003; Zhong et al. 2008)), can obtain better results at the cost of increasing the computational load of the process.

In this paper, a per-pixel, non-recursive, nonpredictive BS approach is described. It has been designed especially for dealing with water background, 


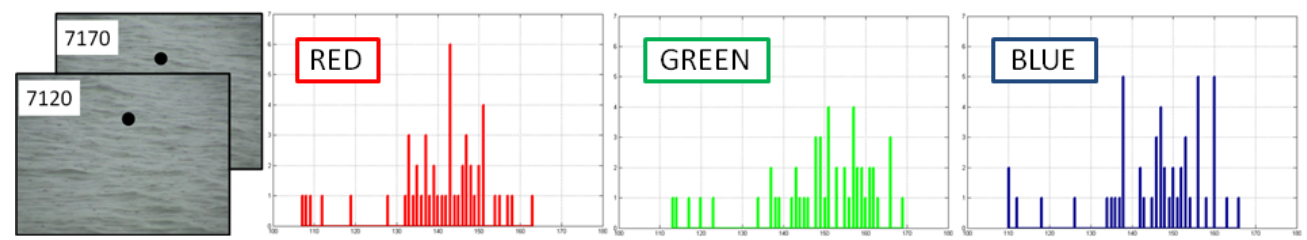

Figure 1: RGB values of a pixel (black dot) from frame 7120 to frame 7170 of Jug sequence.

but can be successfully applied to every scenario. The algorithm is currently in use within a real $24 / 7$ video surveillance system for the control of naval traffic. The main novelties are 1) an on-line clustering algorithm to capture the multimodal nature of the background without maintaining a buffer with the previous frames, 2) a model update mechanism that can detect changes in the background geometry. Quantitative experiments show the advantages of the proposed method over several state-of-the-art algorithms and its real-time performance.

The reminder of the paper is organized as follows. In Section 2 the method is presented and in Section 3 a shadow suppression module is described. The model update process is detailed in Section 4. Experiments demonstrating the effectiveness of the approach are reported in Section 5 and Section 6 provides the conclusions and future work.

\section{The IMBS Method}

The first step of the proposed method is called Independent Multimodal Background Subtraction (IMBS) algorithm and has been designed in order to perform a fast and effective BS. The background model is computed through a per-pixel on-line statistical analysis of a set $L$ of $N$ frames in order to achieve a high computational speed. According to a sampling period $P$, the current frame $I$ is added to $L$, thus becoming a background sample $S_{n}, 1 \leq n \leq N$.

Let $I(t)$ be the $W \times H$ input frame at time $t$, and $F(t)$ the corresponding foreground mask. The background model $\mathfrak{B}$ is a matrix of $H$ rows and $W$ columns. Each element $\mathfrak{B}(i, j)$ of the matrix is a set of tuples $\langle r, g, b, d\rangle$, where $r, g, b$ are RGB values and $d \in[1, N]$ is the number of pixels $S_{n}(i, j)$ associated with those $r, g, b$ values. Modelling each pixel as a tuple has the advantage of capturing the statistical dependences between RGB channels, instead of considering each channel independently.

The method is detailed in Algorithm 1, where $t_{s}$ is the time-stamp of the last processed background sample. IMBS takes as input the sampling period $P$, the number $N$ of background samples to analyse, the minimal number $D$ of occurrences to consider a tuple $\langle r, g, b, d \geq D\rangle$ as a significant background value, and the association threshold $A$ for assigning a pixel to an existing tuple. The procedure RegisterBackground (see Algorithm 2) creates the

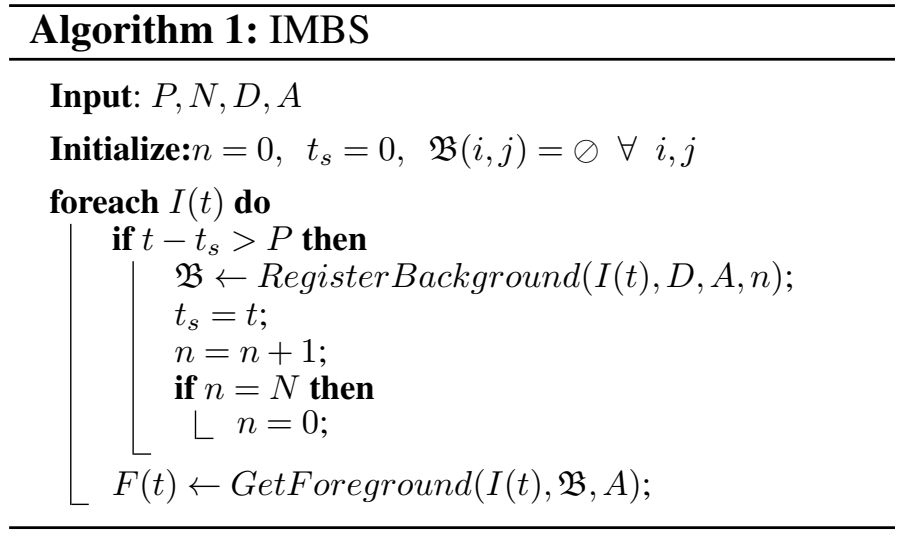

background model, while GetForeground (see Algorithm 3) computes the binary foreground image $F$ representing the output of the process.

Each pixel in a background sample $S_{n}$ is associated with a tuple according to $A$. Once the last sample $S_{N}$ has been processed, if a tuple has a number $d$ of associated samples greater than $D$, then its $r, g, b$ values become a significant background value. Up to $\lfloor N / D\rfloor$ tuples for each element of $\mathfrak{B}$ are considered at the same time allowing for approximating a multimodal probability distribution, that can address the problem of waves, gradual illumination changes, noise in sensor data, and movement of small background elements. Indeed, the adaptive number of tuples for each pixel can model non-regular patterns (Fig. 1) since IMBS do not model the BG with a predefined distribution (e.g., Gaussian), but produces a "discretization" of an unknown distribution.

$F$ is computed according to a thresholding mechanism shown in Algorithm 3, where $|\mathfrak{B}(i, j)|$ denotes the number of sets in $\mathfrak{B}(i, j)$ with $|\mathfrak{B}(i, j)=\oslash|=0$. The use of a set of tuples instead of a single one makes IMBS robust with respect to the choice of the parameter $A$, since a pixel that presents a variation in the RGB values larger than $A$ will be modelled by a set of contiguous tuples.

An example of the 4D background model space for a single pixel is depicted in Fig. 2. The background point is modelled as a set of tuples $\langle r, g, b, d\rangle$ in the RGB color space. Every tuple can be represented graphically by a bin of width $2 A+1$ and height $d$. Bins having height lower than $D$ are not considered as valid background values.

IMBS requires a time $R=N P$ for creating the first background model; then a new background model, 

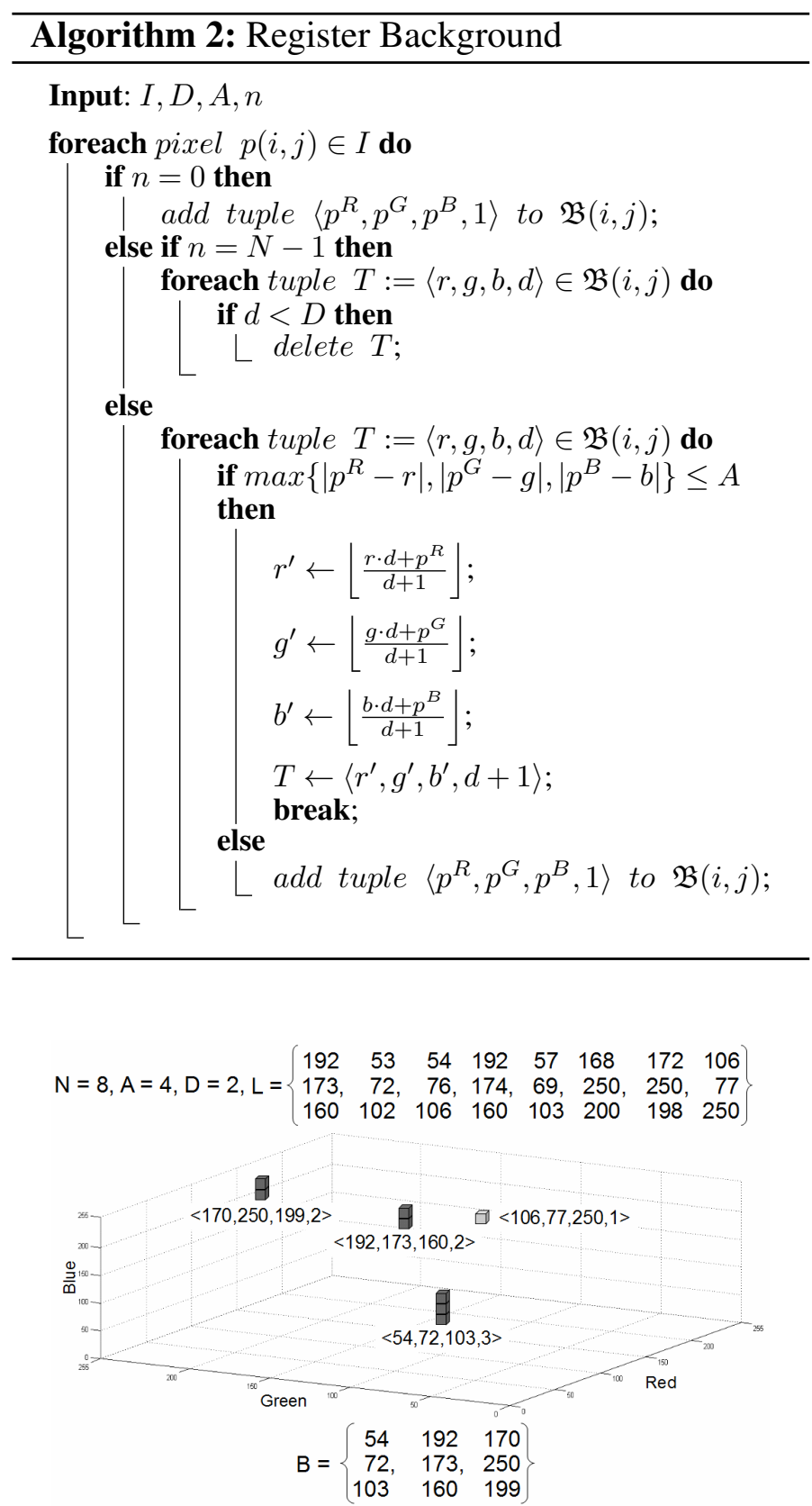

Figure 2: An example of the background model space for a single pixel.

independent from the previous one, is built continuously according to the same refresh time $R$. The independence of each BG model is a key aspect of the algorithm, since it allows to adapt to fast changing environments avoiding error propagation and do not affect the accuracy for slow changing ones.

The on-line model creation mechanism allows for avoiding to store the images belonging to $L$, that is the main drawback of the non-recursive BS techniques (Piccardi 2004). In order to manage illumination changes, $N$ and $P$ values are reduced if the percentage of foreground pixels in $F$ is above a certain threshold (e.g., $50 \%$ ). Moreover, the computational load can be spread over the time interval $P$ until the arrival of the next background sample, thus further increasing the speed of the algorithm.
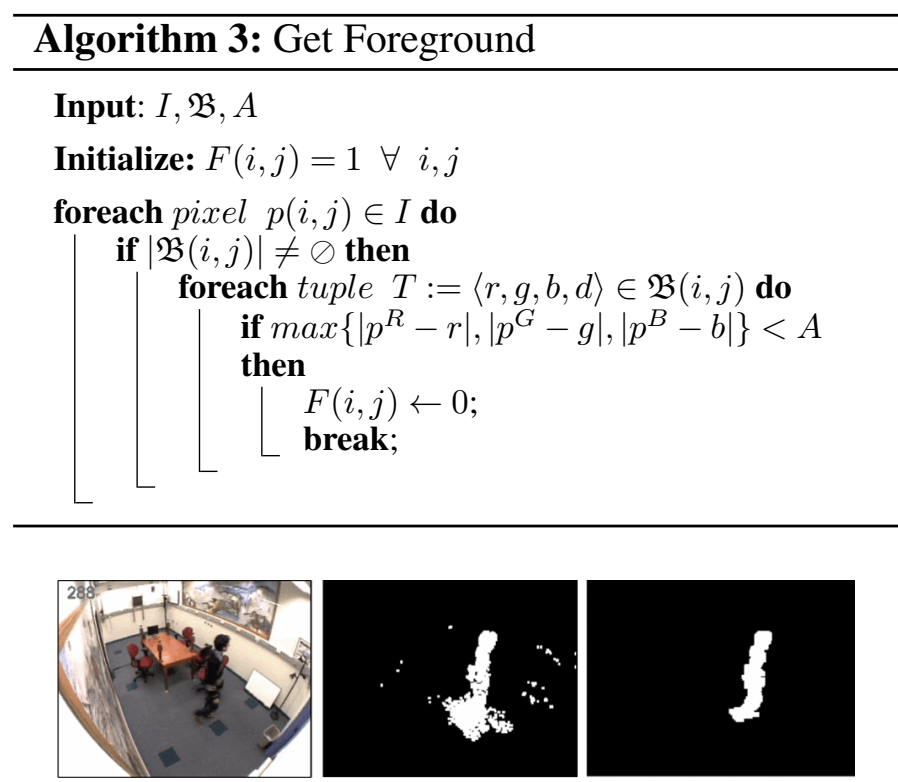

Figure 3: Shadow suppression example. Original frame (left), foreground extraction without shadow removal (center), and with shadow suppression (right).

\section{SHADOW SUPPRESSION}

When BS is adopted to compute the foreground mask, the moving pixels representing both objects and shadows are detected as foreground. In order to deal with the erroneously classified foreground pixels that can deform the detected object shape, a shadow suppression module is required. IMBS adopts a strategy that is a slight modification of the HSV based method proposed by Cucchiara et al. in (Cucchiara et al. 2003).

Let $I^{c}(i, j), \quad c=\{H, S, V\}$ be the HSV color values for the pixel $(i, j)$ of the input frame and $B_{T}^{c}(i, j)$ the HSV values for the tuple $T \in \mathfrak{B}(i, j)$. The shadow mask $M$ value for each foreground point is:

$$
M(i, j)= \begin{cases}1 \quad \text { if } \exists T: \alpha \leq \frac{I^{V}(i, j)}{B_{T}^{V}(i, j)} \leq \beta \wedge \\ & \left|I^{S}(i, j)-B_{T}^{S}(i, j)\right| \leq \tau_{S} \wedge \\ & \left|I^{H}(i, j)-B_{T}^{H}(i, j)\right| \leq \tau_{H} \\ 0 & \text { otherwise }\end{cases}
$$

The parameters $\alpha, \beta, \tau_{S}, \tau_{H}$ are user defined and can be found experimentally. We analysed the sequences from ATON database (ATON ) in order to find the combination of parameters minimizing the error caused by shadows (see Fig. 3). In our OpenCV (OpenCV ) based implementation ${ }^{1}$ we set $\alpha=0.75$, $\beta=1.15, \tau_{S}=30$, and $\tau_{H}=40$. We set $\beta$ to a value slightly greater than 1 to filter out light reflections.

The shadow suppression module is essential for the success of the algorithm. The HSV analysis can effectively remove the errors introduced by a dynamic background, since it is a more stable color space with respect to RGB (Zhao et al. 2002).

\footnotetext{
${ }^{1}$ http://www.dis.uniromal.it/ bloisi/ software/imbs.html
} 


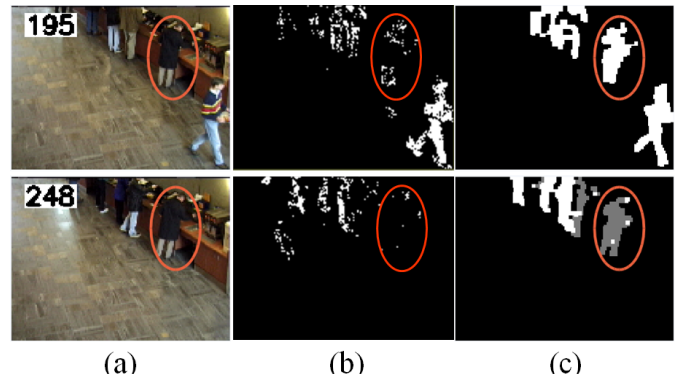

(a)

(b)

(c)

Figure 4: IMBS model update. (a) The target remains in the same position over several frames. (b) A blind update (obtained by OpenCV function CreateGaussianBGModel) includes it in the background model. (c) IMBS model update is able to identify the target as a potential foreground region (grey pixels).

After the removal of the shadow pixels, $F$ can be further refined by exploiting the opening and closing morphological operators. The former is particularly useful for filtering out the noise left by the shadow suppression process, the latter is used to fill internal holes and small gaps.

\section{MODEL UPDATE}

Elgammal et al. in (Elgammal et al. 2000) proposed two alternative strategies to update the background. In selective update, only pixels classified as belonging to the background are updated, while in blind update every pixel in the background model is updated. The selective (or conditional) update improves the detection of the targets since foreground information are not added to the background model, thus solving the problem of ghost observations. However, when using selective updating any incorrect pixel classification produces a persistent error, since the background model will never adapt to it. Blind update does not suffer from this problem since no update decisions are taken, but it has the disadvantage that values not belonging to the background are added to the model.

We propose a different solution that aims to solve the problems of both the selective and blind update. Given the background sample $S_{n}$ and the current foreground mask $F$, if $F(i, j)=1$ and $S_{n}(i, j)$ is associated to a tuple $T$ in the background model under development, then $T$ is labelled as a "foreground tuple". When computing the foreground, if $I(i, j)$ is associated with a foreground tuple, then it is classified as a potential foreground point.

Such a solution allows for identifying regions of the scene representing not moving foreground objects, as in Fig. 4 where a target that remains in the same position over several frames is detected as a potential foreground. The decision about including or not the potential foreground points as part of the background is taken on the basis of a persistence map. If a pixel is classified as potential foreground con-

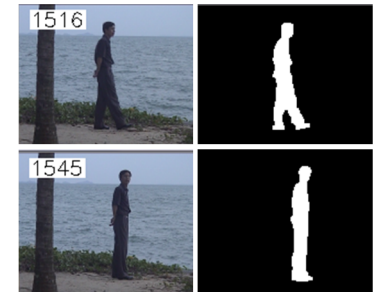

Figure 5: Water surface sequence.

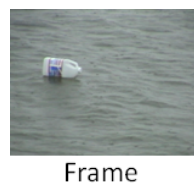

$\# 36$

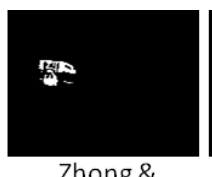

Zhong \& Sclaroff

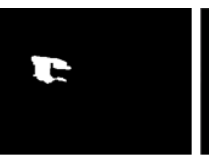

Dalley et al.

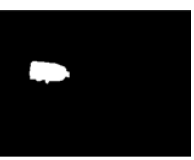

IMBS
Figure 6: Jug sequence.

secutively for a period of time longer than a predefined value (e.g., $R / 3$ ), then it becomes part of the background model. Furthermore, the labelling process provides additional information to higher level modules (e.g., a visual tracking module) helping in reducing ghost observations.

\section{RESULTS}

To test IMBS on water background, we selected two different publicly available sequences: 1) Water surface $^{2}$ : A person walks in front of a water surface with moving waves (see Fig. 5) and 2) $\mathrm{Jug}^{3}$ : A foreground jug floats through the background rippling water (see Fig. 6). IMBS is able to correctly model the background in both the situations, extracting the foreground with great accuracy.

We use also real data coming from a video surveillance system for the control of naval traffic (see Fig. 7). Both people and boats are present in the same scene, allowing the test of the algorithm with different targets. The IMBS conditional update mechanism allows for detecting parked boats (grey pixels in the second row of Fig. 7) before considering them as background. The real data will be made available as part of a public dataset.

To quantitatively compare IMBS with other BS algorithms, we selected the Wallflower sequences (Toyama et al. 1999), a widely used benchmark. In particular, we analysed the following sequences: 1) Moved object (MO), a person enters into a room, makes a phone call and leaves. The phone and the chair are left in a different position; 2) Waving trees (WT), a tree is swaying and a person walks in front of it; 3) Camouflage (C), a person walks in front of a monitor, having rolling interference bars on the screen of the same color of the person's clothing; 4)

\footnotetext{
${ }^{2}$ http://perception.i2r.a-star.edu.sg/bk_ model/bk_index.html

${ }^{3}$ http: //www.cs.bu. edu/groups/ivc/data. php
} 


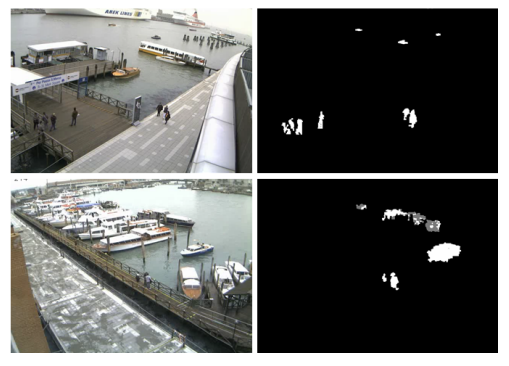

Figure 7: IMBS output with real data.

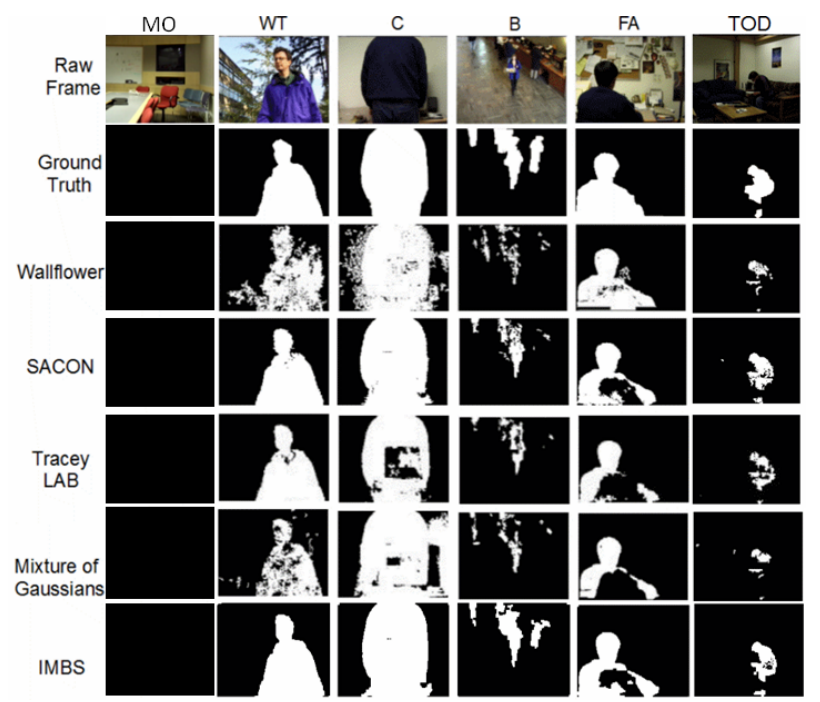

Figure 8: IMBS results (last row) for Wallflower sequences.

Bootstrapping (B), a busy cafeteria where each frame contains foreground objects; 5) Foreground aperture (FA), a person with uniformly coloured shirt wakes up and begins to move; 6) Time of day (TOD), the light in a room is gradually changes from dark to bright. Then, a person enters the room and sits down.

The remaining Wallflower sequence "Light switch" presents a sudden global change in the illumination conditions. In this case, our method fails since it does not include a per-frame module. Anyway, the problem can be faced using the previous computed models and choosing the most appropriate (Ohta 2001).

Fig. 8 shows the IMBS results and the ground truth images for Wallflower dataset as well as the results obtained by applying others methods (Cristani and Murino 2008; Wang and Suter 2006).

In order to make a quantitative comparison with state-of-the-art BS methods, in Table 1 the results are provided in terms of false negatives (the number of foreground points detected as background) and false positives (the number of background points detected as foreground). The results show the effectiveness of the IMBS approach, that performs better than the other methods in terms of total error. Moreover, the computational speed for IMBS on the Wallflower dataset using an Intel Xeon Quad Core $2.26 \mathrm{GHz} 8$ GB RAM CPU is $115 \mathrm{fps}$ (see Table 2), while for the most accurate of the other methods, SACON (Wang

\begin{tabular}{|c|c|c|c|}
\hline Data & Acquisition & Frame Dim. & FPS \\
\hline \hline Wallflower & off-line & $160 \times 120$ & 115 \\
\hline ATON & off-line & $320 \times 240$ & 70 \\
\hline Live 1 & on-line & $320 \times 240$ & 25 \\
\hline Live 2 & on-line & $640 \times 480$ & 14 \\
\hline Live 3 & on-line & $768 \times 576$ & 10 \\
\hline
\end{tabular}

Table 2: IMBS computational speed.

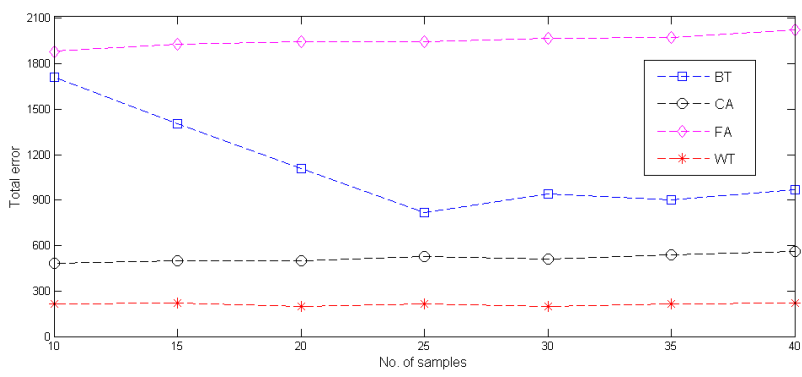

Figure 9: Plot of total error vs. different background sample number.

and Suter 2006), the authors claim a speed of $6 \mathrm{fps}$.

The computational speed of the algorithm has been tested also with on-line data coming from a camera. The results (see Table 2) show the real-time performance of the proposed approach.

For all the test sequences, IMBS parameters have been set as: $A=5, N=30, D=2$, and $P=1000$ $m s$. We investigate the influence of the parameter $N$ on the results (see Fig. 9): Given the same refresh time $R=30 \mathrm{sec}$, various $N$ and $P$ values are used. For all the sequences, except for the sequence $B$, there are similar total error values even with a limited number of background samples. Since $B$ is the sequence with the most dynamical background, there is a significant increase in the total error if $N$ is small. However, starting from $N=25$, the total error value becomes stable, as for the other sequences.

\section{CONCLUSIONS}

In this paper, a fast and robust background subtraction algorithm has been proposed and quantitatively compared to several state-of-the-art methods using a well-known benchmark dataset. Thanks to an on-line clustering algorithm to create the model and a novel conditional update mechanism, the method is able to achieve good accuracy while maintaining real-time performances. As future work, we intend to investigate the use of the HSV color space in building the background model, and to add per-region and perframe modules to the algorithm.

\section{REFERENCES}

ATON. http://cvrr.ucsd.edu/aton/testbed.

Cheung, S. and C. Kamath (2004). Robust techniques for background subtraction in urban traffic video. In Visual Comm. and Image Proc., Volume 5308, pp. 


\begin{tabular}{|c|c|c|c|c|c|c|c|c|}
\hline Method & Err. & MO & $\mathbf{W T}$ & $\mathbf{C}$ & $\mathbf{B}$ & FA & TOD & Tot.E. \\
\hline \multirow{3}{*}{ Wallflower (Toyama et al. 1999) } & f. neg. & $\overline{0}$ & 877 & 229 & 2025 & $\overline{\overline{320}}$ & $\overline{9961}$ & \multirow{3}{*}{10156} \\
\hline & f. pos. & 0 & 1999 & 2706 & 365 & 649 & 25 & \\
\hline & tot. e. & 0 & 2876 & 2935 & 2390 & 969 & 986 & \\
\hline \multirow{3}{*}{ SACON (Wang and Suter 2006) } & f. neg. & 0 & 41 & 47 & 1150 & 1508 & 236 & \multirow{3}{*}{4467} \\
\hline & f. pos. & 0 & 230 & 462 & 125 & 521 & 147 & \\
\hline & tot. e. & 0 & 271 & 509 & 1275 & 2029 & 383 & \\
\hline \multirow{3}{*}{$\begin{array}{c}\text { Tracey LAB } \\
\text { LP (Kottow et al. 2004) }\end{array}$} & f. neg. & 0 & 191 & 1998 & 1974 & 2403 & 772 & \multirow{3}{*}{8046} \\
\hline & f. pos. & 1 & 136 & 69 & 92 & 356 & 54 & \\
\hline & tot. e. & 1 & 327 & 2067 & 2066 & 2759 & 826 & \\
\hline \multirow{3}{*}{$\begin{array}{c}\text { Bayesian } \\
\text { Decision (Nakai 1995) }\end{array}$} & f. neg. & 0 & 629 & 1538 & 2143 & 2511 & 1018 & \multirow{3}{*}{15603} \\
\hline & f. pos. & 0 & 334 & 2130 & 2764 & 1974 & 562 & \\
\hline & tot. e. & 0 & 963 & 3688 & 4907 & 4485 & 1580 & \\
\hline \multirow{3}{*}{$\begin{array}{c}\text { Eigen- } \\
\text { background (Oliver et al. 2000) }\end{array}$} & f. neg. & 0 & 1027 & 350 & 304 & 2441 & 879 & \multirow{3}{*}{16353} \\
\hline & f. pos. & 1065 & 2057 & 1548 & 6129 & 537 & 16 & \\
\hline & tot. e. & 1065 & 3084 & 1898 & 6433 & 2978 & 895 & \\
\hline \multirow{3}{*}{$\begin{array}{c}\text { Mixture of } \\
\text { Gaussians (Stauffer and Grimson 1999) }\end{array}$} & f. neg. & 0 & 1323 & 398 & 1874 & 2442 & 1008 & \multirow{3}{*}{11251} \\
\hline & f. pos. & 0 & 341 & 3098 & 217 & 530 & 20 & \\
\hline & tot. e. & 0 & 1664 & 3496 & 2091 & 2972 & 1028 & \\
\hline \multirow{3}{*}{ IMBS } & f. neg. & $\mathbf{0}$ & 23 & 83 & 388 & 1438 & 294 & \multirow{3}{*}{3696} \\
\hline & f. pos. & $\mathbf{0}$ & 152 & 345 & 301 & 564 & 117 & \\
\hline & tot. e. & $\mathbf{0}$ & 175 & 428 & 689 & 2002 & 411 & \\
\hline
\end{tabular}

Table 1: A comparison of IBMS with state-of-the-art methods on Wallflower dataset.

$881-892$.

Cristani, M. and V. Murino (2008). Background subtraction with adaptive spatio-temporal neighbourhood analysis. In VISAPP, Volume 2, pp. 484-489.

Cucchiara, R., C. Grana, M. Piccardi, and A. Prati (2003). Detecting moving objects, ghosts, and shadows in video streams. PAMI 25(10), 1337-1342.

Dalley, G., J. Migdal, and W. Grimson (2008). Background subtraction for temporally irregular dynamic textures. In IEEE Workshop on Applications of Computer Vision, pp. 1-7.

Doretto, G., A. Chiuso, Y. N. Wu, and S. Soatto (2003). Dynamic textures. IJCV 51(2), 91-109.

Elgammal, A. M., D. Harwood, and L. S. Davis (2000). Non-parametric model for background subtraction. In ECCV, pp. 751-767.

Heikkila, M. and M. Pietikainen (2006). A texturebased method for modeling the background and detecting moving objects. PAMI 28(4), 657-662.

Kottow, D., M. Koppen, and J. Ruiz del Solar (2004). A background maintenance model in the spatial-range domain. In SMVP, pp. 141-152.

Mittal, A. and N. Paragios (2004). Motion-based background subtraction using adaptive kernel density estimation. In CVPR, pp. 302-309.

Nakai, H. (1995). Non-parameterized bayes decision method for moving object detection. In Asian Conf. Comp. Vis., pp. 447-451.

Ohta, N. (2001). A statistical approach to background subtraction for surveillance systems. IEEE Int. Conf. on Computer Vision 2, 481-486.

Oliver, N. M., B. Rosario, and A. P. Pentland (2000). A bayesian computer vision system for modeling human interactions. PAMI 22(8), 831-843.

OpenCV. http://opencv.willowgarage.com.

Piccardi, M. (2004). Background subtraction techniques: a review. In Proc. of the IEEE Int. Conf. on Systems, Man \& Cybernetics, pp. 3099-3104.

Sheikh, Y. and M. Shah (2005). Bayesian object detection in dynamic scenes. In CVPR, pp. 74-79.

Stauffer, C. and W. Grimson (1999). Adaptive background mixture models for real-time tracking. CVPR 2, 246-252.

Tavakkoli, A. Nicolescu, M. and G. Bebis (2006). Robust recursive learning for foreground region detection in videos with quasi-stationary backgrounds. In $I C P R$, pp. 315-318.

Toyama, K., J. Krumm, B. Brumitt, and B. Meyers (1999). Wallflower: principles and practice of background maintenance. In ICCV, Volume 1, pp. 255261.

Wang, H. and D. Suter (2006). Background subtraction based on a robust consensus method. In ICPR, pp. 223-226.

Zhao, M., J. Bu, and C. Chen (2002). Robust background subtraction in hsv color space. In SPIE: Multimedia Systems and Applications, pp. 325-332.

Zhong, B., H. Yao, S. Shan, X. Chen, and W. Gao (2008). Hierarchical background subtraction using local pixel clustering. In $I C P R$, pp. 1-4.

Zhong, J. and S. Sclaroff (2003). Segmenting foreground objects from a dynamic textured background via a robust kalman filter. In $I C C V$, pp. 4450. 